\title{
High-Speed Tracking of Moving Markers During Radiotherapy Using a CMOS Active Pixel Sensor
}

\author{
JPF Osmond ${ }^{1}$, G Lupica ${ }^{2}$, EJ Harris ${ }^{1}, \mathrm{H} \mathrm{Zin}^{1}$, NM Allinson ${ }^{3}$, PM Evans ${ }^{1}$ \\ ${ }^{1}$ The Institute of Cancer Research, Sutton, United Kingdom, \\ ${ }^{2}$ The University of Sheffield, Sheffield, United Kingdom, \\ ${ }^{3}$ The University of Lincoln, Lincoln, United Kingdom
}

Purpose/Objective(s): In order to minimize the dose delivered to healthy tissue near a moving tumor during radiotherapy it is first necessary to accurately measure tumor position as a function of time. For example, a portal imager can be used to detect surrogate markers implanted around the tumor in order to track its motion with a moving collimator. Lung tumors can move at up to $30 \mathrm{~mm} / \mathrm{s}$, requiring a sampling rate of 30 frames/s to achieve mm accuracy. However the passive a-Si Flat Panel Imagers (FPIs) available with current linear accelerators operate at 2-10 frames/s, significantly slower than the required rate. Furthermore a-Si FPIs provide low image quality at their fastest frame rates and are susceptible to damage by the treatment beam, requiring replacement every 1 - 2 years. Emerging CMOS active pixel sensors use an addressable and partial read-out architecture to achieve significantly improved frame-rates relative to their passive counterparts. They are also capable of higher resolution, image quality and radiation-hardness. This study investigates the feasibility of using a CMOS APS to quickly and accurately track radio-opaque markers during radiotherapy.

Materials/Methods: A custom CMOS imaging system was designed and constructed in collaboration with the MI3 consortium. The performance of this system was characterized and compared with an aSi FPI. Four cylindrical gold markers of diameter 0.8 to $2 \mathrm{~mm}$ and length $8 \mathrm{~mm}$ were positioned on a motion-platform and moved according to the Lujan approximation to respiratory motion. Images were acquired using the megavoltage treatment beam at a range of frame and dose rates. The success rate of an automatic detection routine, absolute mean-error from the expected position and contrastto-noise ratio of the marker images were then evaluated as a function of marker size, marker speed, frame rate and dose rate.

Results: The CMOS imager was found to offer improved resolution and signal-to-noise than the standard a-Si FPI at a comparable dose. The long integration time of the FPI resulted in marker images being too blurred to detect. The CMOS was able to detect the three largest markers $100 \%$ of the time and estimate their position to within $0.3 \mathrm{~mm}$ at $150-300 \mathrm{MU} / \mathrm{min}$ and $20-50 \mathrm{frame} / \mathrm{s}$. However success rate declined with decreasing dose or frame rate.

Conclusions: A CMOS megavoltage imaging system was found to offer superior signal-noise and resolution than the standard a-Si FPI. Furthermore the high speed of CMOS provided sub mm tracking of moving markers at a clinically acceptable dose rate and marker size

Author Disclosure: J.P.F. Osmond: None. G. Lupica: None. E.J. Harris: None. H. Zin: None. N.M. Allinson: None. P.M. Evans: None. 